\title{
Characterisation of Acinetobacter with special reference to carbapenem resistance and biofilm formation
}

\author{
Pattanaik A. ${ }^{1}$, G.S Banashankari \\ ${ }^{1}$ Dr. Amrita Pattanaik, ${ }^{2}$ Dr. Banashankari G.S., both authors are affiliated with Department of Microbiology, M.S \\ Ramaiah Medical College and Teaching Hospital, Bangalore, Karnataka, India. E-mail: banashankarigs@gmail.com \\ Corresponding Author: Dr. Banashankari G.S., Department of Microbiology, M.S Ramaiah Medical College and \\ Teaching Hospital, Bangalore, Karnataka, India. E-mail: banashankarigs@gmail.com
}

\begin{abstract}
Background: Acinetobacter species cause hospital outbreaks and are often multidrug resistant. A wide range of resistance determinants make them successful nosocomial pathogens. In the present study, authors have identified and speciated Acinetobacter from various clinical specimens by a simplified phenotypic identification scheme determined their antibiotic susceptibility pattern focussing on Carbapenem resistance and have also evaluated their biofilm producing ability. Method: Clinical samples were screened for Acinetobacter species and isolates were speciated. Antibiogram was determined by performing Kirby-Bauer disc diffusion method. Isolates resistant to Carbapenems were subjected to Modified Hodge Test (MHT) and Meropenem-EDTA Combined Disc Test (CDT). These isolates were further evaluated for their biofilm forming ability by the Microtitre Plate Method. Results: Out of 174 isolates, the species most frequently isolated was Acinetobacter calcoaceticus-baumannii complex (ACB) (89.1\%). 70.1\% isolates were resistant to Carbapenems, of which $45.1 \%$ were MHT positive and $73.8 \%$ were CDT positive. $63.7 \%$ of the isolates were biofilm producers. Conclusion: Simple identification schemes and antimicrobial susceptibility testing are cost effective and require fewer resources. Screening for Carbapenem resistance can help avoid unnecessary use of broad-spectrum antibiotics and thereby prevent treatment failure. Biofilms lead to decreased penetrability of antibiotics and make managing infections a clinical challenge. Further research is required to have a better understanding of the mechanism of biofilm formation and its implication in drug resistance.
\end{abstract}

Keywords: Acinetobacter species, Carbapenem resistance, Biofilm formation

\section{Introduction}

Acinetobacter recently has become the centre of focus for the clinicians worldwide because of its pathogenic potential [1]. These strains are commonly isolated from the hospital environment and from colonised or infected individuals [2]. The wide range of resistance determinants along with environment resilience makes them successful nosocomial pathogens [3]. These pathogens show resistance to major antibiotic classes [4].

Acinetobacter causes a wide variety of nosocomial infections like bacteremia, pneumonia (particularly ventilator associated pneumonia), urinary tract infection, and secondary meningitis [5]. According to the published data, Acinetobater baumannii ventilatorassociated pneumonia and bloodstream infections have been associated with a high degree of mortality and morbidity [6].

Manuscript received: $5^{\text {th }}$ June 2019

Reviewed: $14^{\text {th }}$ June 2019

Author Corrected: $20^{\text {th }}$ June 2019

Accepted for Publication: $25^{\text {th }}$ June 2019
Characterisation of Acinetobacter is effectively done using molecular techniques. They have been divided into various genomic species [6]. It has helped in understanding the epidemiology as well as in formulating appropriate preventive and treatment protocols [2]. In laboratories which are unable to perform the advanced molecular studies, certain simplified phenotypic tests based on growth characteristics and metabolic requirement of the organism aid in speciation.

These tests are not reliable (lack sensitivity and reproducibility), cause confusion and can only help in the presumptive identification [5]. In this study, authors have attempted to speciate the clinical isolates of Acinetobacter using a few simplified phenotypic tests.

Clinical isolates of Acinetobacter have shown widespread resistance to the major groups of antibiotics [7]. Carbapenem resistance is dreaded as it is associated with high mortality because of delays in administration 
of effective treatment. There is also the issue of limited availability of treatment options in the developing countries. Newer antibiotics capable of replacing Carbapenems are not likely to become available in the near future. The strains resistant to Carbapenems spreadquickly in the healthcare settings and in the community $[1,8]$.

Acinetobacter frequently causes infections associated with medical devices like the endotracheal tube, central venous catheters, Foley's catheter, etc. A well-known virulence factor in such infections is formation of biofilms. Biofilms on abiotic surfaces may facilitate their survival in the environment [1,9]. Increased synthesis of exopolysaccharide (EPS) and the

\section{Original Research Article}

development of antibiotic resistance are important properties of biofilm forming organisms. It can be assumed that increased production of EPS in Acinetobacter might be creating a protective environment which in turn might beleading to poor antibiotic penetration and development of resistance.

Also, it so appears that there may be some differences in the cellular physiology of cells within the biofilm that may also result in increased resistance to the drugs [1, 10].

The present study evaluates the frequency and extent of biofilm formation and the antibiotic resistance in all the isolated strains.

\section{Methods}

Setting: The study was conducted at M.S Ramaiah Medical College and Teaching Hospital, Bangalore.

Duration and type of study: This was a prospective study done on clinical samples like pus, urine, blood, respiratory specimen, peritoneal fluid, cerebrospinal fluid received for culture and sensitivity in the department of Microbiology at M.S Ramaiah Medical College and Teaching Hospital during the period of 1 year (January 2016 to December 2016).

Sampling method: All the samples sent for culture and sensitivity to the microbiology laboratory over the period of one year were included in the study.

Sample size calculation: Sample size calculation was based on a previous study conducted by Mindolli et al [2] where Acinetobacter species were isolated from $4.25 \%$ of positive cultures. In the present study, considering an absolute precision of $3 \%$ and confidence level of $95 \%$, sample size was calculated to be 174 with the help of n-master software.

Inclusion criteria: Clinical samples like urine, pus, respiratory specimen, peritoneal fluid, blood, cerebrospinal fluid were screened for Acinetobacter species in a period of 1 year (January 2016 to December 2016)

Exclusion criteria: No exclusion criteria envisaged.

Data analysis: IBM SPSS version 20 software was used for analysing the data.

Ethical consideration and permission: Ethical clearance was obtained from the institutional ethical committee.

Laboratory procedures- All specimens received were subjected to direct microscopy and culture: The specimens were inoculated on to Mac Conkey agar and Blood agar. In case of urine, the specimens were inoculated on to Cysteine lactose electrolyte deficient (CLED). The inoculated media were incubated at $37^{\circ} \mathrm{C}$ overnight and observed for growth. Culture plates were then examined for specific colonies (Figure 1) [11].

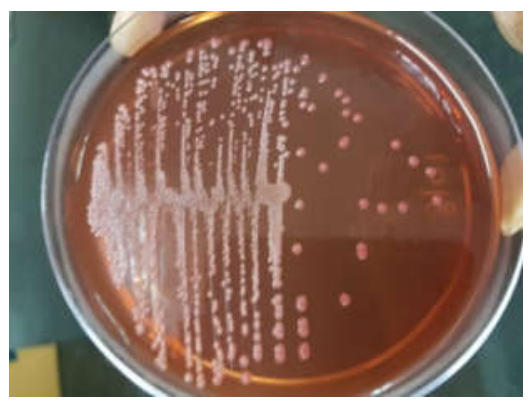

Figure-1: Mac Conkey plate showing pale non lactose fermenting colonies 


\section{Original Research Article}

Identification of Acinetobacter- Colonies on the primary culture plate were subjected to Gram's stain, hanging drop technique for motility, tests for production of enzymes, tests for utilisation of substrate, tests for metabolism of proteins and amino acids and tests for utilisation of carbohydrates [12].

Speciation of Acinetobacter was done on the basis of: Hemolysis on blood agar, Growth at $42^{\circ} \mathrm{C}$, Oxidation fermentation test, Arginine dihydrolase test, Malonate utilisation, Gelatin liquefaction [3].

Antibiotic susceptibility testing- Acinetobacter isolates were subjected to antibiotic susceptibility testing by employing Kirby Bauer disc diffusion technique according to CLSI 2015 guidelines [13]. In the present study, the susceptibility was tested against Ceftazidime, Cefepime, Ciprofloxacin, Imipenem, Meropenem, Gentamicin, Amikacin, Tobramycin, Piperacillin, Ampicillin-Sulbactam and Piperacillin-Tazobactam.

Isolates resistant to Imipenem and Meropenem or any one of them was further tested with Imipenem and Meropenem E (Epsilometric) strips. Results were interpreted by the zone of inhibition. MIC value was the value at which the zone intersected the strip.

The resistant isolates were further screened for Carbapenemase and Metallo beta lactamase (MBL) production by Modified Hodge test (MHT) and Meropenem - EDTA Combined Disc Test (CDT) test respectively $[1,14]$.

Modified Hodge Test: Escherichia coli (ATCC 25922) colonies were inoculated into normal saline and incubated at $37^{\circ} \mathrm{C}$ for 6 hours to obtain an optical density of $0.5 \mathrm{McFarland}$ turbidity standards. This suspension was then diluted by a factor of 10. A lawn culture of this diluted suspension was done on the Mueller Hinton Agar (MHA) plate and was allowed to stand at room temperature for a period of 5 minutes. A $10 \mathrm{mcg}$ Meropenem disc was placed at the centre and the test organism was streaked in a straight line from the edge of the disc to the edge of the plate. The plate was then incubated at $35 \pm 2^{\circ} \mathrm{C}$ in ambient air for 16-22 hours. A distorted zone of inhibition or clover leaf indentation at the intersection of the test organism and E.coli ATCC 25922 within the zone of Meropenem susceptibility disc (Figure 2A) was interpreted as positive result $[1,15]$.

Meropenem- EDTA Combined Disc Test (CDT)- A 0.5 M EDTA with a $\mathrm{pH}$ of 8 was prepared and sterilized by autoclaving. On a $10 \mathrm{mcg}$ Meropenem disc, $10 \mu \mathrm{l}$ this EDTA solution was put. Few colonies of test organism were inoculated in the nutrient broth and incubated at $37^{\circ} \mathrm{C}$ for $4-6$ hours and the suspension turbidity was matched to 0.5 McFarland turbidity standards. Lawn culture of this suspension of test organism was done on MHA. One 10 $\mu \mathrm{g}$ Meropenem disk was placed on MHA plate. An EDTA impregnated Meropenem disc was also placed on the same MHA plate at the distance of $20-25 \mathrm{~mm}$ from centre to centre. The plate was incubated at $37^{\circ} \mathrm{c}$ for $16-18$ hours. An increase in zone size of $\geq 7 \mathrm{~mm}$ around the Meropenem-EDTA disc compared to Meropenem without EDTA (Figure 2B) was recorded as an MBL producing strain $[1,16]$.

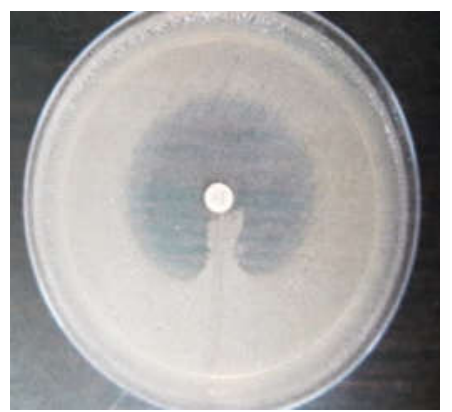

Figure-2A: Modified Hodge Test

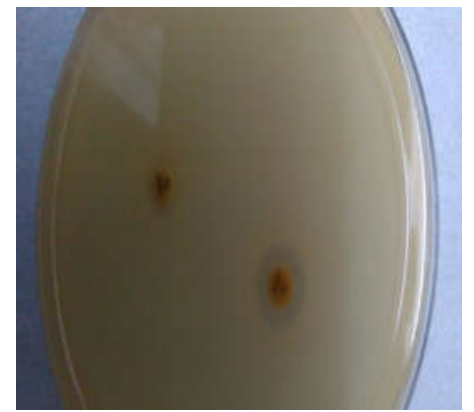

Figure-2B: Combined Disc Test

\section{Assessment of biofilm production in the strains}

Microtitreplate method: Each isolate was grown overnight in trypticase soy broth (TSB) with $0.25 \%$ glucose at $37^{\circ} \mathrm{C}$. The overnight growth was diluted by a factor of 40 in TSB- $0.25 \%$ glucose. $200 \mathrm{mcl}$ of cell suspension was inoculated in sterile 96 well polystyrene microtitre plates. After 24 hours of incubation, the wells were gently washed three times with $200 \mathrm{mcl}$ of phosphate buffered saline (PBS), dried in an inverted position and stained with $1 \%$ crystal violet for $15 \mathrm{~min}$ 


\section{Original Research Article}

(Figure 3). The wells were rinsed again in 200 microlitre of ethanol-acetone $(80: 20 \mathrm{v} / \mathrm{v})$ to solubilise crystal violet. Using a microplate reader the optical density at $620 \mathrm{~nm}$ (OD 620) was determined. The average optical density of each assay performed in triplicate was considered.

For determining biofilm formation the following OD values were considered:

Non-biofilm producer: OD620 $<0.25(\mathrm{ODc})$

Weak biofilm producer: $0.25(\mathrm{ODc}) \leq \mathrm{OD} 620<0.50(2 \mathrm{ODc})$

Medium biofilm producer: $0.50(2 \mathrm{ODc}) \leq \mathrm{OD} 620<0.75(3 \mathrm{ODc})$

Strong biofilm producer: $0.75(3 \mathrm{ODc}) \leq$ OD620

The value 0.25 was $3 \mathrm{SD}$ above the mean optical density of a clean microtitre plate well stained by the above method [1, 17].

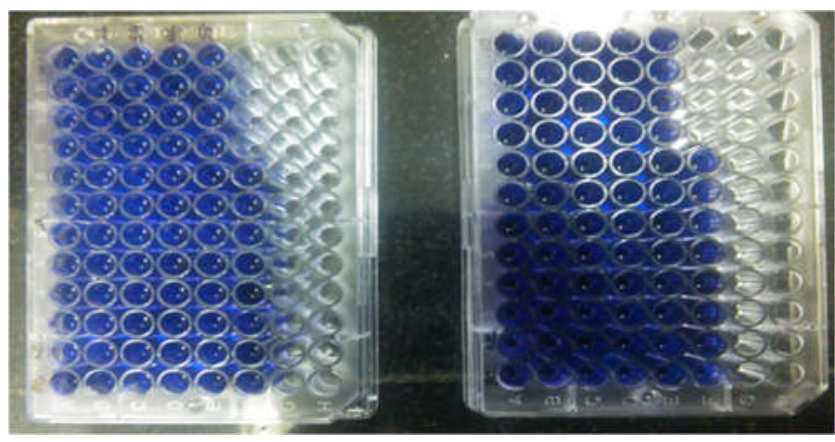

Figure-3: Microtitre Plate Method for Biofilm Detection

\section{Results}

Out of the 174 isolates, $110(63.2 \%)$ were from the different Intensive Care Units (ICU) and 64 (36.8\%) were from the general wards and outpatient departments. The organism was isolated from various clinical samples. $54(30.5 \%)$ isolates were from the pus samples, 47 (27.6\%) were from the ET secretions, 33 (19\%) were from blood, 18 (10.3\%) were from the tissue specimens. Next in frequency were catheter tips $(4 \%)$ followed by urine $(2.9 \%)$, ascitic fluid $(1.7 \%)$ and sputum (1.7\%). There were 2 isolates from the pleural fluid (1.1\%), 1 each from cerebrospinal fluid and ear swab (0.6\%) (Figure 4).

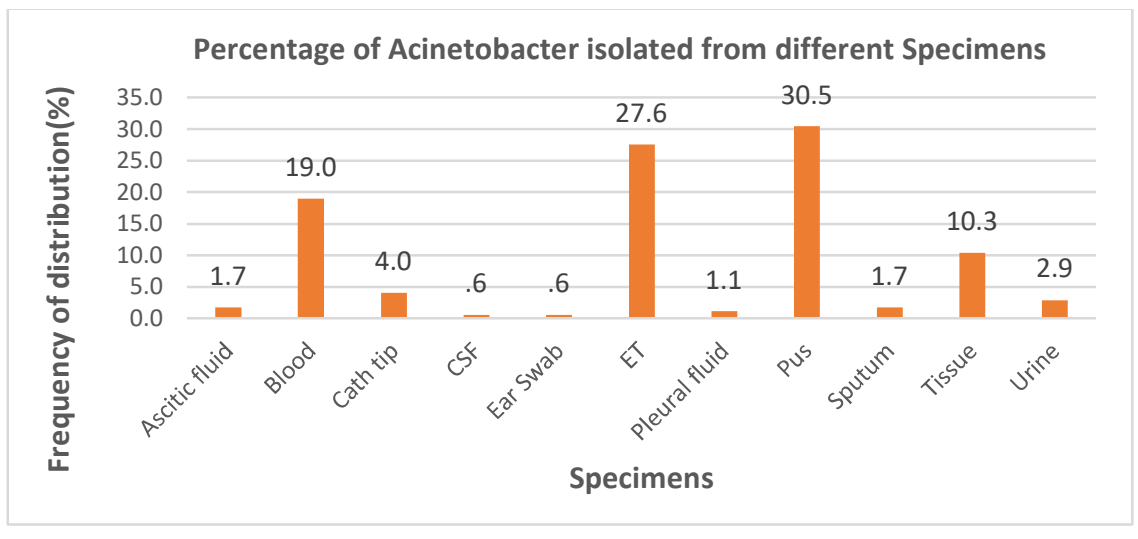

Figure-4: Bar graph representing the frequency of distribution of Acinetobacter species in different clinical specimens.

$30.5 \%$ of Acinetobacter isolates were from pus, followed by $27.6 \%$ isolation from ET and $19 \%$ from blood. The species most frequently isolated was ACB complex, constituting about $89.1 \%$ (155) of all isolates following which was Acinetobacter lwoffii, constituting 8\% (14) of the isolates. There were 2 isolates (1.1\%) each of Acinetobacter junii and Acinetobacter hemolyticus. Only one isolate $(0.6 \%)$ of Acinetobacter radioresistens was found in the specimens (Figure 5). 
Original Research Article

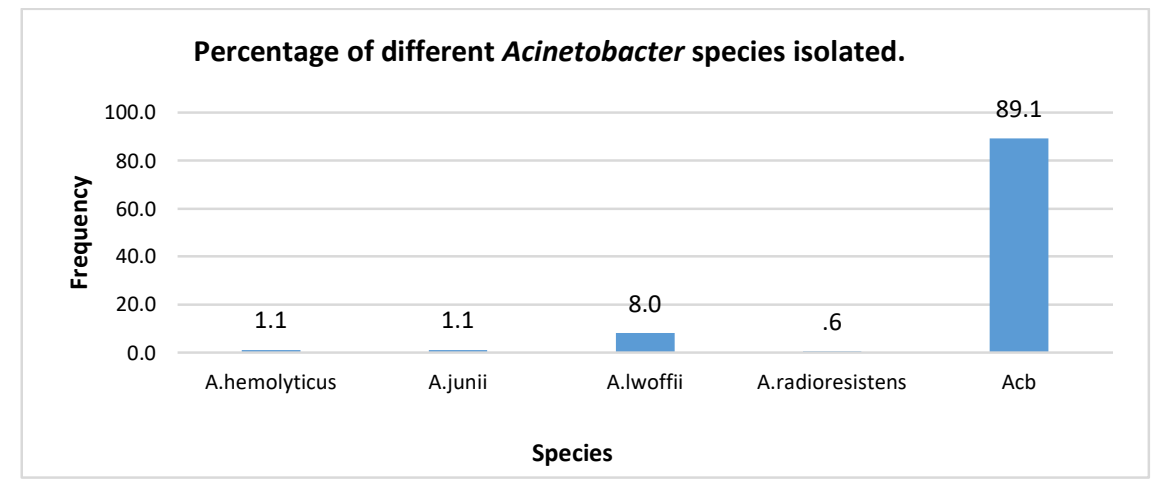

Figure-5: Frequency of isolation of different Acinetobacter species.

Acinetobacter calcoaceticus-baumannii complex (ACB) was the most commonly isolated species $(89.1 \%)$ followed by Acinetobacter lwoffii (8\%)

Table-1: Distribution of Acinetobacter species in different clinical specimens.

\begin{tabular}{|c|c|c|c|c|c|c|c|c|c|c|c|c|}
\hline 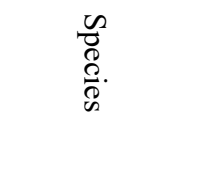 & $\vec{E}$ & $\stackrel{\text { M }}{\rightarrow}$ & $\begin{array}{l}\overrightarrow{\mid} \\
\overrightarrow{0} \\
\overrightarrow{0}\end{array}$ & : & $\begin{array}{l}\tilde{Q} \\
\stackrel{\Xi}{E} \\
\bar{E}\end{array}$ & $\begin{array}{l}\text { f } \\
\text { 至 }\end{array}$ & 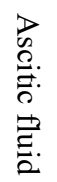 & 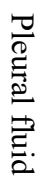 & 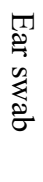 & $\begin{array}{l}\frac{\varpi}{\circ} \\
\vdots \\
\triangleright\end{array}$ & 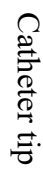 & $\stackrel{-1}{\stackrel{0}{ٍ ٍ ~}}$ \\
\hline ACB complex & $\begin{array}{l}4 \\
9\end{array}$ & 45 & 15 & 5 & 2 & 1 & 2 & 1 & 1 & 28 & 6 & 155 \\
\hline A.lwoffi & 4 & 1 & 2 & 0 & 0 & 0 & 1 & 1 & 0 & 4 & 1 & 14 \\
\hline A.hemolyticus & 0 & 0 & 1 & 0 & 0 & 0 & 0 & 0 & 0 & 1 & 0 & 2 \\
\hline A.junii & 1 & 1 & 0 & 0 & 0 & 0 & 0 & 0 & 0 & 0 & 0 & 2 \\
\hline $\begin{array}{l}\text { A.radio } \\
\text { resistens }\end{array}$ & 0 & 0 & 0 & 0 & 1 & 0 & 0 & 0 & 0 & 0 & 0 & 1 \\
\hline
\end{tabular}

Table-2: Antibiotic sensitivity of Acinetobacter species isolated

\begin{tabular}{|c|c|c|c|c|}
\hline $\begin{array}{c}\text { SL. } \\
\text { No }\end{array}$ & Antibiotics (disc strength) & $\begin{array}{c}\text { Resistant- No (\%) } \\
\text { of isolates }\end{array}$ & $\begin{array}{c}\text { Intermediately } \\
\text { Sensitive -No (\%) }\end{array}$ & $\begin{array}{c}\text { Sensitive- No (\%) } \\
\text { of isolates }\end{array}$ \\
\hline 1 & Ceftazidime (30mcg) & $149(85.6 \%)$ & - & $25(14.4 \%)$ \\
\hline 2 & Gentamicin (10mcg) & $123(70.7 \%)$ & $7(4.0 \%)$ & $44(25.3 \%)$ \\
\hline 3 & Cefepime & $149(85.6 \%)$ & - & $25(14.4 \%)$ \\
\hline 4 & Ciprofloxacin & $149(85.6 \%)$ & - & $25(14.4 \%)$ \\
\hline 5 & Meropenem (10mcg) & $119(68.4 \%)$ & $3(1.8 \%)$ & $52(29.8 \%)$ \\
\hline 6 & Imipenem & $112(64.4 \%)$ & $6(3.4 \%)$ & $56(32.2 \%)$ \\
\hline 7 & Amikacin (30mcg) & $114(65.5 \%)$ & $3(1.7 \%)$ & $57(32.8 \%)$ \\
\hline 8 & Piperacillin & $148(85.1 \%)$ & - & $26(14.9 \%)$ \\
\hline 9 & Piperacillin-Tazobactam & $148(85.1 \%)$ & - & $26(14.9 \%)$ \\
\hline 10 & Ampicillin-Sulbactam & $125(71.8 \%)$ & $5(2.9 \%)$ & $44(25.3 \%)$ \\
\hline 11 & Tobramycin & $134(77 \%)$ & - & $40(23 \%)$ \\
\hline
\end{tabular}

Carbapenem resistance and detection of carbapenemase production

Out of the 174 isolates, 122 (70.1\%) were resistant to the Carbapenems as determined by the Epsilometer test. For the detection of carbapenemase production, Modified Hodge Test (MHT) and Combined Disc Test (CDT) were performed on the 122 isolates. MHT was positive for $45.1 \%$ and CDT was positive for $73.8 \%$ of the isolates (Figure 6 ). 


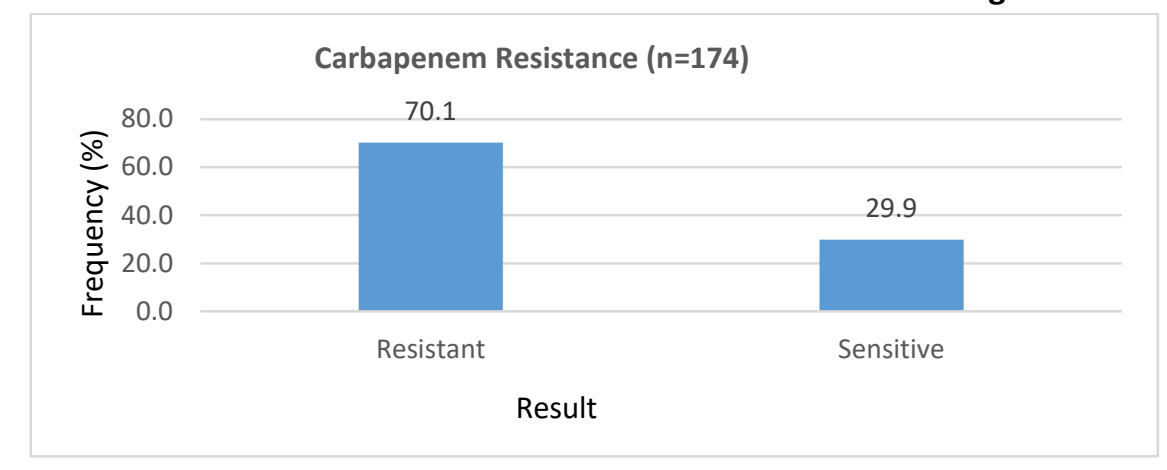

Figure-6: Frequency distribution of Carbapenem resistance in Acinetobacter species. $70.1 \%$ of the Acinetobacter isolates were resistant to Carbapenems.

Biofilm production was evaluated in all the 174 isolates using the microtitre plate method. $63.7 \%$ of the isolates were biofilm producers and the rest $36.3 \%$ were biofilm non producers (Figure 7 ).

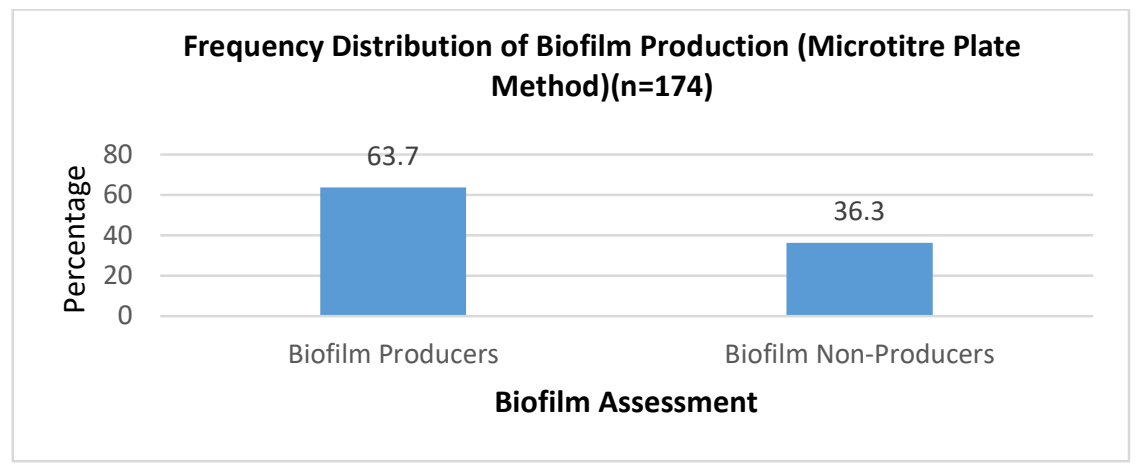

Figure-7: Frequency distribution of biofilm production in Acinetobacter species. About $63.7 \%$ of the Acinetobacter isolates were biofilm producers.

\section{Discussion}

Acinetobacter species have emerged as important nosocomial pathogens. These multidrug resistant organisms are often associated with life threatening infections. A. baumannii especially has a tendency towards cross transmission, mostly in ICUs, where there are numerous outbreaks. Acinetobacter infection, in the present study, was more common in patients over 40 years of age. Out of the 174 isolates studied here, $110(63.2 \%)$ were from the different Intensive Care Units (ICU) and $64(36.8 \%)$ were from the general wards and outpatient departments. This finding is similar to the data published by Raina et al [18]. Most of the patients in the ICU had some underlying condition like chronic obstructive pulmonary disease (COPD), bronchial asthma, acute respiratory distress syndrome and other predisposing factors such as diabetes mellitus, chronic renal failure, liver cirrhosis and immunosuppression. Most of them were on prior antibiotic therapy as well. This was similar to an observation made by Vincent et al [19] and Lee et al [20]. It can be thus concluded that prior use of antibiotics, underlying infections and invasive procedures like catheterisation, intravenous fluids or ventilator support were important risk factors for development of the development of nosocomial Acinetobacter infection $[3,18,21,22]$.

In the current study, Acinetobacter was isolated from various clinical samples. $54(30.5 \%)$ isolates were from the pus samples, 47 (27.6\%) were from the ET secretions, $33(19 \%)$ were from blood, 18 (10.3\%) were from the tissue specimens. Next in frequency, were catheter tips (4\%) followed by urine (2.9\%), ascitic fluid (1.7\%) and sputum (1.7\%). There were 2 isolates from the pleural fluid (1.1\%), 1 each from cerebrospinal fluid and ear swab $(0.6 \%)$. The single isolate from the cerebrospinal fluid was shunt fluid from a patient who had undergone neurosurgery. In a similar study by Raina et al [18], isolation of Acinetobacter was maximum from tips (43.4\%), followed by pus (26.4\%) and blood (17\%). Two (3.8\%) Acinetobacter isolates were from CSF. In another study by Lahiri et al[22], majority of isolates were found in urine samples (51.3\%) and Oberoi A et al [23] reported maximum isolation rate of Acinetobacter from pus samples $(86.2 \%)$ which was similar to the present study. Most of the pus isolates were from patients having cellulitis and wound infections. 


\section{Original Research Article}

In the present study, the species most frequently isolated was ACB complex, constituting about $89.1 \%$ (155) of all isolates following which was Acinetobacter lwoffii, constituting 8\% (14) of the isolates. There were 2 isolates (1.1\%) each of Acinetobacter junii and Acinetobacter hemolyticus. Only one isolate (0.6\%) of Acinetobacter radioresistens was found in the specimens and that was from a sputum sample of a patient diagnosed with bronchopneumonia. Predominance of A. baumannii (90.6\%) was reported by Raina et al [18], followed by A. lwoffii and A. haemolyticus showed an isolation rate of $5.7 \%$ and $3.8 \%$ respectively. Singlaet al [24] have reported an isolation rate of $74.6 \%$ for $A$. baumannii followed by A.lwoffii (24.3\%).

In $95.4 \%$ cases, monomicrobial Acinetobacter infection was seen and in rest $5.6 \%$ cases it was polymicrobial. E. coli was the most common associated organism. In a study conducted by Joshi SG et al [25] in 2006, monomicrobial infections accounted for $71.2 \%$ and $28.8 \%$ were polymicrobial. Mindolli et al [3] in 2010 reported polymicrobial infections in $87.5 \%$ cases and E.coli was the commonest organism isolated along with Acinetobacter. They also speculated that in polymicrobial infections, Acinetobacter species were more resistant to antibiotics and were associated with high morbidity in the patients. Any statistically significant association was not observed in the present study.

The resistance rates of different antibiotics for the isolates were - Ceftazidime (85.6\%), Cefepime (85.6\%), Ciprofloxacin (85.6\%), Piperacillin and Piperacillin-Tazobactam (85.1\%), Ampicillin-Sulbactam (74.7\%), Tobramycin (77\%), Gentamicin (74.7\%), Amikacin (67.2\%), Imipenem (64.4\%), Meropenem (70.1\%). Minimal inhibitory concentration of Tigecycline tested for all the isolates showed a susceptibility rate of $96 \%$. Raina et al[18] reported high levels of resistance for Ampicillin-sulbactam (96\%), Ampicillin (94\%), Aztreonam (94\%), Cefuroxime (92\%), ceftazidime (91\%). Significant levels of resistance were also recorded for Piperacillin-Tazobactam (83\%), Cefipime (83\%), Amikacin (83\%), Trimethoprim- sulfamethoxazole (83\%) and Levofloxacin (81\%). Taneja et al [26], in their study have reported that the resistance of Acinetobacter to gentamicin, amikacin and ciprofloxacin was $79.5 \%, 73.2 \%$ and $72.8 \%$ respectively. Shareek et al [27] reported that $75 \%$ of the strains were resistant to carbapenems, $85 \%$ were resistant to $\beta$-lactams and 72 $80 \%$ of the strains were resistant to amikacin, ciprofloxacin and cotrimoxazole. Similar findings have been reported by Raina et al [18]. Even in the present study a high level of carbapenem resistance (70.1\%) was seen. Carbapenem resistance is emerging as a huge threat not only in ICUs but also in the wards. A study by Jaggi et al [28] showed resistance to Carbapenem in $89 \%$ of the isolates. An analysis of the resistance pattern for various antibiotics used against Acinetobacter infections in ICUs and wards showed a shift of the resistance pattern more towards the ICU isolates. Resistance patterns among nosocomial pathogens vary widely from location to location because of which a surveillance of nosocomial pathogens for resistograms is needed for appropriate selection for empiric therapy. It can also be a primary pointer for the emergence of an outbreak. Detection of resistance in a particular pattern may suggest a currently occurring epidemic in the hospital or the persistence of a particular strain of the bacteria [29]. Strains of ACB-complex were more resistant to all the antibiotics tested as compared to A.lwoffii, A.haemolyticus, A.junii and A.radioresistens.

Carbapenems are generally the last resort in the treatment of life threatening infections caused by multidrug resistant Acinetobacter isolates. Emergence of Carbapenem hydrolysing $\beta$-lactmases of Ambler class B (MBLs) and class D (Oxacillinases/CHDLs) have been proven to be the most important mechanism of carbapenem resistance and thus have caused difficulty in the treatment. Simple and accurate tests are needed to detect MBL producers. Meropenem-EDTA combined disc test and Modified Hodge test have been used in this study for MBL detection. Though CLSI does not advocate the use of MHT for detection of Carbapenemase production in non-fermenting gram negative bacilli, several authors have found MHT with Imipenem, EDTA and $\mathrm{ZnSO}_{4}$ as a useful screening test for Carbapenemase production $[30,31]$.

Table-3: A comparative analysis of the tests performed by different authors.

\begin{tabular}{|c|c|c|c|c|}
\hline SL.NO & Author \& Year & Strains Tested & CDT & MHT \\
\hline 1. & Irfan S et al [32], 2011 & 100 & $96.6 \%$ & - \\
\hline 2. & Amudhan SM et al [30], 2011 & 116 & $79.3 \%$ & $97.4 \%$ \\
\hline 3. & John S et al [33], 2011 & 242 & - & $14.8 \%$ \\
\hline 4. & Shivprasad A et al [31], 2014 & 168 & $81.18 \%$ & $100 \%$ \\
\hline 5. & Agrawal R et al [16], 2015 & 56 & $87.5 \%$ & $84 \%$ \\
\hline 6. & Das NK et al [34], 2016 & 94 & $76.59 \%$ & - \\
\hline 7. & Present Study & 122 & $73.8 \%$ & $45.1 \%$ \\
\hline
\end{tabular}




\section{Original Research Article}

Screening for carbapenem resistance and detecting carbapenemase and MBL producers among Acinetobacter isolates in resource limited setting helps to avoid unnecessary use of broad spectrum antibiotics and thereby prevent treatment failures and development of resistance. The global spread of multidrug resistant Acinetobacter spp. is a major challenge in the clinical setting. Drugs such as Colistin, Polymyxin B, Tigecycline and Doripenem, are being tried for treating such infections [35].

Biofilm production was assessed in all the 174 isolates. $63.7 \%$ of the isolates (included the strong, moderate and the weak biofilm producers) were biofilm producers and the rest $36.3 \%$ were biofilm non producers. In a similar study by Bala et al [36], 62.5\% were biofilm producers among the clinical Acinetobacter isolates. Similar occurrence of $63 \%$ and $62 \%$ biofilm formers have also been reported by Rodriguez et al [37], and Rao et al [38], respectively.

There was a significant association seen between Carbapenem resistance and biofilm formation $(p=0.013)$. This was in concordance with studies conducted by Abdi et al [39] and Rao et al [38]. Biofilms on surfaces result in decreased penetrability of antibiotics and makes managing infections a clinical challenge. In a similar study, Rao et al [38] and Rong et al [40] reported a significant association between multidrug resistance and biofilm. The study by Rao et al [38] showed that the presence of blaPER-1 was more critical for cell adhesion than the formation of bacterial biofilms on abiotic surfaces.

\section{Conclusion}

Traditional typing methods like phenotyping and antibiogram typing have an advantage over genotyping as they are easily available in all clinical microbiology laboratories. Simple identification schemes and antimicrobial susceptibility testing are cost effective.

Screening for carbapenem resistance and detecting carbapenemase and MBL producers among Acinetobacter isolates in resource limited setting helps to avoid unnecessary use of broad spectrum antibiotics and thereby prevent treatment failures and development of resistance.

Biofilms on surfaces result in decreased penetrability of antibiotics and make managing infections a clinical challenge. Further research should concentrate on the genetic and molecular mechanisms associated with the formation of biofilm.

Understanding biofilm formation and the genetic basis for control of this process will be instrumental in developing new strategies for dealing with infections caused by these opportunistic and often multi-drug resistant nosocomial pathogens.Novel treatment strategies such as phage therapy, quorum-sensing inhibition, and induced biofilm-dispersion have to be further worked upon.

\section{Author contributions}

- Dr. Banashankari GS: Designed and guided the study. Contributed in the data analysis. Corrected and finalised the manuscript.

- Dr. Amrita Pattanaik: Performed the required tests. Organised and analysed the data. Prepared the manuscript.

\section{What does this study add to the existing knowledge?}

This study not only gives an overview of the clinical burden of the nosocomial pathogen, Acinetobacter but also analyses the antibiotic resistance of the organism to the most widely used group of broad spectrum antibiotics - Carbapenems.

Biofilm formation, an important virulence factor of the organism has been studied with a special focus on it probable association with antibiotic resistance.

Findings: Nil; Conflict of Interest: None initiated Permission from IRB: Yes

\section{References}

1. Pattanaik A, GS Banashankari. Assessment of Biofilm Production in Carbapenem Resistant Acinetobacter Species Isolated from Different Clinical Specimens. Journal of Medical Science and Clinical Research 2017;5(10):29103-10.DOI: https://dx.doi.org / 10. $18535 / \mathrm{jmscr} / \mathrm{v} 5 \mathrm{i} 10.108$

2. Mindolli PB, Salmani MP, Vishwanath G, Hanumanthapa AR. Identification and speciation of Acinetobacter and their antimimicrobial susceptibility testing. Al Ameen J Med Sci. 2010;3:3459.

3. Salmani MP, Mindolli PB. Speciation and Antimicrobial Susceptibility Pattern of Acinetobacter from Clinical Isolates in a Tertiary Care Centre. 2015;4 (10) : 973-976.

4. Perez F, Hujer AM, Hujer KM, et al. Global challenge of multidrug-resistant Acinetobacter baumannii. Antimicrob Agents Chemother. 2007; 51 (10) : 3471-84. 


\section{Original Research Article}

5. Bergogne-Berezin E, Friedman H, Bendinelli M, editors. Acinetobacter Biology and Pathogenesis: Springer; New York 2008.

6. Bergogne-Bérézin E, Towner KJ. Acinetobacter spp. as nosocomial pathogens: microbiological, clinical, and epidemiological features. ClinMicrobiol Rev. 1996; 9 (2): 148-65.

7. Vaneechoutte M, Dijkshoorn L, Nemec A, Kämpfer P, Wauters G. Acinetobacter, Chryseobacterium, Moraxella, and Other Nonfermentative Gram-Negative Rods. In: Versalovic J, Carroll K, Funke G, Jorgensen J, Landry M, Warnock D (editors). Manual of Clinical Microbiology, 10th Edition. ASM Press; Washington 2011: 714-738.

8. Towner KJ. Clinical importance and antibiotic resistance of Acinetobacter spp. Proceedings of a symposium held on 4-5 November 1996 at Eilat, Israel. J Med Microbiol.1997;46(9):721-46.DOI:10.1099/0022 2615-46-9-721

9. European Centre for Disease Prevention and Control. Rapid risk assessment: Carbapenem- resistant Enterobacteriaceae. ECDC: 2016. Available from: http://ecdc. europa.eu /en/publications/Publications /carbapenemresistant-enterobacteriaceae-risk-assessment-april-2016. pdf

10. Rodríguez-Baño J, Martí S, Soto $\mathrm{S}$, et al. Biofilm formation in Acinetobacter baumannii: associated features and clinical implications. ClinMicrobiol Infect. 2008;14(3):276-8.DOI:10.1111/j.1469-0691.2007. 01916. x. Epub 2008 Jan 10.

11. Collee JG, Duguid JP, Fraser AG, Marmion BP, Simmons A. Laboratory strategy in the diagnosis of infective syndromes. In Collee JG, Marmion BP, Fraser AG, Simmons A. Editors. Mackie and McCartney practical medical microbiology. $14^{\text {th }}$ ed. New York; Church Livingstone publication; 1996: 53-94.

12. The Nonfermentative Gram-Negative Bacilli. In: Winn W, Allen S, Janda W, Koneman E, Procop G, Schreckenberger $\mathrm{P}$, et al, editors. Colour Atlas and Textbook of Diagnostic Microbiology, 6th edition: Lippincott, Williams and Wilkins Publications; Philadelphia, USA 2006: 353-355

13. CLSI. 2015. Performance standards for antimicrobial susceptibility testing; twenty-second informational supplements. CLSI document M100-S25. Wayne PA:Clinical and Laboratory Standards Institute.
14. Sivasankari S, Senthamarai, Anitha C, Sastry A, Bhatt S, Kumudhavathi M S, et al. Phenotypic detection of carbapenem resistance in clinical isolates of Acinetobacter baumanii in Kanchipuram.Int J Appl Biol Pharm Technol 2014;5(1):50-53.

15. Modified Hodge Test for carbapenemase detection inEnterobacteriaceae.www.cdc.gov/HAI/pdfs/labSetting $\mathrm{s} /$ HodgeTest_Carbapenemase_Enterobacteriaceae

16. Agrawal R, Sumana MN, Kishore A, Kulkarni M. Simple Method for Detection of Metallo - $\beta$-Lactamase Among Gram Negative Isolates. Online J Health Allied Sci. 2015;14(3):6.

17. Badave GK, Kulkarni D. Biofilm Producing Multidrug Resistant Acinetobacter baumannii: An Emerging Challenge. J Clin Diagn Res. 2015;9(1): DC08- 10. DOI: 10. 7860/ JCDR/2015/ 11014. 5398.

18. Raina D, Sharma N, Mahawal BS, Khanduri A, Pandita A. Speciation and antibiotic resistance pattern of Acinetobacter species in a tertiary care hospital in Uttarakhand. International Journal of Medical Research \& Health Sciences 2016; 5(4): 89-96.

19. Vincent JL, Rello J, Marshall J, Silva E, Anzueto A, Martin CD et al. International study of the prevalence and outcomes of infection in intensive care units. JAMA.2009;302(21):2323-9. DOI: 10.1001/jama. 2009. 1754.

20. Lee SO, Kim NJ, Choi SH, Kim TH, Chung JW, Woo JH, et al. Risk factors for acquisition of imipenemresistant Acinetobacter baumannii: a case-control study. Antimicrob Agents Chemother. 2004;48(1):224-8. DOI: 10. 1128/aac.48.1.224-228.2004

21. Lone R, Shah A, Kadri SM, Lone S, Faisal S. Nosocomial multi-drug-resistant Acinetobacter infections- Clinical findings, risk factors and demographic characteristics. Bangladesh J Med Microbiol. 2009; 3(01) 34-38.

22. Lahiri K, Mani NS, Purai SS. Acinetobacter species as nosocomial pathogen: Clinical significance and antimicrobial sensitivity. Med J Armed Forces India. 2004;60(1):7-10.

23. Oberoi A, Aggarwal A, Lal M. A decade of an underestimated nosocomal pathogen- Acinetobacter in a tertiary care hospital in Punjab. JK Science 2009; 11(1): 24-26. 


\section{Original Research Article}

24. Singla P, Sikka R, Deep A, Seema, Chaudhary U. Pattern of antimicrobial resistance in clinical isolates of Acinetobacter species at a tertiary level health care facility in northern India. J Evol Med Dental Sci 2013;2 (2):159-165.

25. Joshi SG, Litake GM, Satpute MG, Telang NV, Ghole VS, Niphadkar KB. Clinical and demographic features of infections caused by Acinetobacter species. Indian J Med Sci 2006;60(9):357-360.

26. Taneja N, Singh G, Singh M, Sharma M. Emergence of tigecycline \&colistin resistant Acinetobacter baumanii in patients with complicated urinary tract infections in north India. Indian $\mathrm{J}$ Med Res. 2011;133:681-4.

27. Shareek PS, Sureshkumar D, Ramgopalakrishnan, Ramasubramanian V, Ghafur K, Thirunarayanan MA. Antibiotic Sensitivity Pattern of Blood Isolates of Acinetobacter species in a Tertiary Care Hospital: A RetrospectiveAnalysis.Am.J.Infect.Dis.2012;8(1):65-69

28. Jaggi N, Sissodia P and Sharma L. Acinetobacter baumannii isolates: Epidemiology, antibiogram and nosocomial status studied over a 25 month period in a tertiary care hospital in India. Proceedings of the International Conference on Prevention and Infection Control, Jun. 29-Jul. 2, Geneva, Switzerland. 2011.

29. Prashanth K, Badrinath S. In vitro susceptibility pattern of Acinetobacter species to commonly used cephalosporins, quinolones, and aminoglycosides. Indian J Med Microbiol. 2004;22(2):97-103.

30. Amudhan SM, Sekar U, Arunagiri K, Sekar B. OXA beta-lactamase-mediated carbapenem resistance in Acinetobacter baumannii. Indian J Med Microbiol. 2011; 29(3):269-74. DOI: 10.4103/0255-0857.83911.

31. Shivaprasad A, Antony B, Shenoy P. Comparative evaluation of four phenotypic tests for detection of metallo-beta-lactamase and carbapenemase production in Acinetobacter baumannii. J Clin Diagn Res. 2014; 8 (5):DC05-DC08.DOI: 10.7860/JCDR/ 2014/6447. 4317

32. Irfan $\mathrm{S}$, Zafar $\mathrm{A}$, Guhar $\mathrm{D}$, Ahsan $\mathrm{T}$, Hasan $\mathrm{R}$. Metallo-beta-lactamase-producing clinical isolates of Acinetobacter species and Pseudomonas aeruginosa from intensive care unit patients of a tertiary care hospital. Indian J Med Microbiol. 2008;26(3):243-5. DOI: $10.4103 / 0255-0857.42035$

33. John S, Balagurunathan R. Metallo beta lactamase producing Pseudomonas aeruginosa and Acinetobacter baumannii. Indian J Med Microbiol. 2011;29(3):302-4. DOI: $10.4103 / 0255-0857.83918$.

34. Das NK, Grover N, Sriram R, Kumar M, Dudhat VL, Prasanna S. Prevalence of Carbapenem Resistance and Comparison between Different Phenotypic Methods for Detection of Metallo-B-Lactamases in Gram Negative Non-Fermentative Bacteria in the Acute Wards of a Tertiary Care Centre. Int J Curr Microbiol App Sci 2016; 5(5):109-119. DOI: http://dx.doi.org/10. 20546/ijcmas.2016.505.012

35. Manchanda V, Sanchaita S, Singh N. Multidrug resistant acinetobacter. J Glob Infect Dis. 2010;2(3): 291-304. DOI: 10.4103/0974-777X.68538.

36. Bala M, Gupte S, Aggarwal P, Kaur M, Manhas A. Biofilm producing multidrug resistant Acinetobacter species from a tertiary care hospital: a therapeutic challenge. 2016; 4(7):3024-6. DOI: http://dx.doi.org /10. 18203/2320-6012.ijrms20161997

37. Rodríguez-Baño J, Martí S, Soto S, et al. Biofilm formation in Acinetobacter baumannii: associated features and clinical implications. ClinMicrobiol Infect. 2008; 14(3): 276-8.DOI:10.1111/j.1469-0691. 2007. 01916.x. Epub 2008 Jan 10.

38. Rao RS, Karthika RU, Singh SP, et al. Correlation between biofilm production and multiple drug resistance in imipenem resistant clinical isolates of Acinetobacter baumannii. Indian J Med Microbiol. 2008;26 (4):333-7. DOI: 10.4103/0255-0857.43566

39. Abdi-Ali A, Hendiani S, Mohammadi P, Gharavi S. Assessment of biofilm formation and resistance to imipenem and ciprofloxacin among clinical isolates of Acinetobacter baumannii in Tehran. Jundishapur $\mathrm{J}$ Microbiol 2014;7(1):e8606. DOI: 10.5812/jjm.8606

40. Rong D, Chun G, Dan H, Ting-ting X, Yan Q. The correlation study on antimicrobial resistance and biofilm related genes in the clinical isolates of Acinetobacter baumannii. Chin Crit Care Med 2013; 25(8): 493-94. DOI: 10.3760/cma. j. issn.2095-4352. 2013. 08.011.

\section{How to cite this article?}

Pattanaik A, G.S Banashankari. Characterisation of Acinetobacter with special reference to carbapenem resistance and biofilm formation. Trop J Path Micro 2019;5(6):386-395. doi:10.17511/jopm.2019.i06.09. 